\title{
Direct Measurement of the $g$-Factor of Composite Fermions
}

\author{
F. Schulze-Wischeler*, E. Mariani ${ }^{\dagger}$, F. Hohls* and R. J. Haug* \\ *Institut für Festkörperphysik, Universität Hannover, Appelstraße 2, D-30167 Hannover, Germany \\ ${ }^{\dagger}$ Department of Condensed Matter Physics, The Weizmann Institute of Science, 76100 Rehovot, Israel
}

\begin{abstract}
The activation gap $\Delta$ of the fractional quantum Hall states at constant fillings $v=2 / 3$ and $2 / 5$ has been measured as a function of the perpendicular magnetic field $B$. A linear dependence of $\Delta$ on $B$ is observed while approaching the spin polarization transition, yielding a direct measurement of the $g$-factor of Composite Fermions.
\end{abstract}

In recent years, the ever improving mobility of the samples allowed the observation of the fractional quantum Hall $(\mathrm{FQH})$ effect in the low magnetic field regime. Here, the spin degree of freedom plays a crucial role, and FQH states with partial (or vanishing) spin polarization have been directly observed [1-4].

The theoretical understanding of the $\mathrm{FQH}$ effect has been deepened by the introduction of Composite Fermions (CF) [5], quasiparticles made of one electron bound to an even number of magnetic flux quanta. As far as the properties of $\mathrm{CF}$ are concerned, the effective mass $m^{*}$ has attracted a lot of interest while much less has been said about their spin-related behaviour [6]. Only the filling factor dependence of the product $m^{*} g[3,8]$ was determined from observations of the spin-polarization transitions $[4,7]$, but no direct measurement of the CF $g$ factor was accomplished yet.

In this work we present an experimental analysis of the activation gap $\Delta$ at constant filling $v=2 / 3$ and $v=2 / 5$ as a function of the purely perpendicular magnetic field $B$. We observed a linear magnetic field scaling of $\Delta$ while approaching the spin-polarization transition which yields a direct measurement of the $\mathrm{CF} g$-factor [9].

Composite Fermions around half filling of the lowest electronic Landau level (LL) are created by attaching two magnetic flux quanta to each electron. Thus, $\mathrm{CF}$ feel an effective average magnetic field $B^{*}=B(1-2 v)$ in an external magnetic field $B$. The CF filling factor $p$ is related to the electronic one by $v=p /(2 p \pm 1)$, mapping the principal sequence of the electronic $\mathrm{FQH}$ states into an integer quantum Hall effect of $\mathrm{CF}$. The $\mathrm{CF}$ cyclotron gap at fixed $p$ scales as $\hbar \omega_{\mathrm{c}}^{*} \equiv \hbar e B^{*} / m^{*} \propto 1 /(2 p \pm$ 1) $e^{2} / \varepsilon l$ since the Coulomb term $e^{2} / \varepsilon l(\approx 51 \sqrt{B[\mathrm{~T}]} \mathrm{K})$ is the only relevant energy scale for electrons in the lowest LL [10] ( $\varepsilon \approx 12.8$ is the dielectric constant for GaAs and $l=\sqrt{\hbar / e B}$ is the magnetic length). Such a scaling can be obtained by assuming an effective CF mass $m^{*} \propto \sqrt{B}$. In real experiments disorder and finite thickness corrections tend to reduce the activation gaps. Since the CF flux attachment does not couple to the electronic spin degree of freedom, the Zeeman term can easily be included and it depends only on $B$. Thus, the energies of spin-up/down $(s= \pm 1 / 2)$ CF LL are

$$
E_{n p s}(B)=\left(n+\frac{1}{2}\right) \hbar \omega_{\mathrm{c}}^{*}(p, B)+s g \mu_{\mathrm{B}} B
$$

with $\mu_{\mathrm{B}}=0.67 \mathrm{~K} / \mathrm{T}$ the Bohr magneton.

The zero-temperature ground state at a given $B$ is obtained by occupying the lowest $p$ spin-split CF LL. Due to the different $B$-scaling of the cyclotron and Zeeman terms in Eq. (1), CF LL with opposite spins cross. The transitions between differently polarized ground states are then given by the crossings between CF LL at the Fermi energy. We can define the "slope" $S_{n p s}(B)=$ $\partial_{B} E_{n p s}(B)$. It is then easy to check that, independently on the value of $m^{*}$,

$$
\left.\left|S_{n p \uparrow}\left(B_{n n^{\prime}}\right)-S_{n^{\prime} p \downarrow}\left(B_{n n^{\prime}}\right)\right| \equiv \partial_{B} \Delta\right|_{B_{n n^{\prime}}}=\frac{1}{2}|g| \mu_{\mathrm{B}},
$$

with $B_{n n^{\prime}}$ the magnetic field where the two levels $E_{n p \uparrow}$ and $E_{n^{\prime} p \downarrow}$ cross. The energy gap $\Delta$ approaching the transition is therefore linear and its slope given by Eq. (2) depends uniquely on the $\mathrm{CF} g$-factor. The model above neglects CF-CF interactions which however become relevant only very close to the spin-polarization transition $[4,13]$.

In what follows we will describe our activation gap measurement for fixed $v=2 / 3$ and 2/5 (i.e. $p=2$ ) and directly deduce the $\mathrm{CF} g$-factor from the linear scaling described in the model above.

The two-dimensional electron system used in our experiments was realized in an AlGaAs/GaAsheterostructure and the carrier density was modulated by 


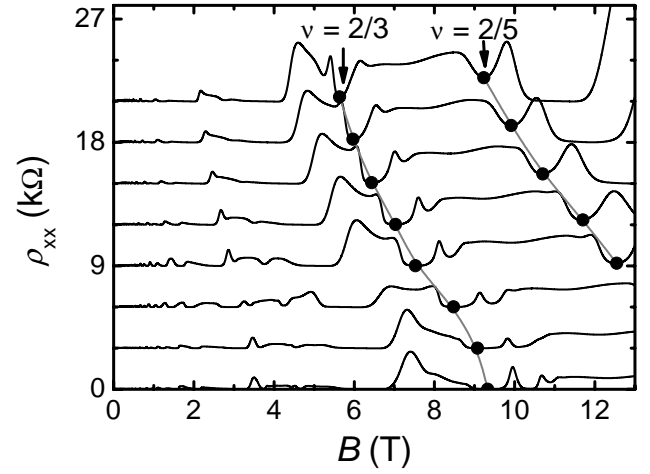

FIGURE 1. Resistivity $\rho_{\mathrm{xx}}(B)$ of all measured electron densities (shifted by $3 \mathrm{k} \Omega$ each for clarity). At $v=2 / 3$ and $v=2 / 5$ the activation measurements are performed.

illumination. The base carrier density and mobility are $n_{e}=0.89 \cdot 10^{15} \mathrm{~m}^{-2}$ and $\mu_{e}=102 \mathrm{~m}^{2} / \mathrm{Vs}$. With maximal illumination $n_{e}=1.50 \cdot 10^{15} \mathrm{~m}^{-2}$ and $\mu_{e}=193 \mathrm{~m}^{2} / \mathrm{Vs}$ are achieved.

An infrared LED allows to change the carrier density $n_{e}$ using the persistent photoconductivity. For different $n_{e}$ we measure the resistivity $\rho_{\mathrm{xx}}$ as function of $B$, which allows to determine the carrier density and mobility. Fig. 1 shows $\rho_{\mathrm{xx}}$ of all achieved densities.

For each density we measured the temperature dependence of the resistivity $\rho_{\mathrm{xx}}(T)$ while fixing the magnetic field corresponding to filling factors $v=2 / 3$ and $2 / 5$. We observe activated transport $\rho_{\mathrm{xx}} \propto \exp (-\Delta / 2 T)$ with $\Delta$ the activation gap. From the fits to this equation, we extract $\Delta$ at $v=2 / 3$ and $v=2 / 5$ for different magnetic fields. The dependence of $\Delta$ on $B$ is shown in Fig. 2 . A remarkably linear behaviour is observed over a large magnetic field range, in agreement with the theoretical

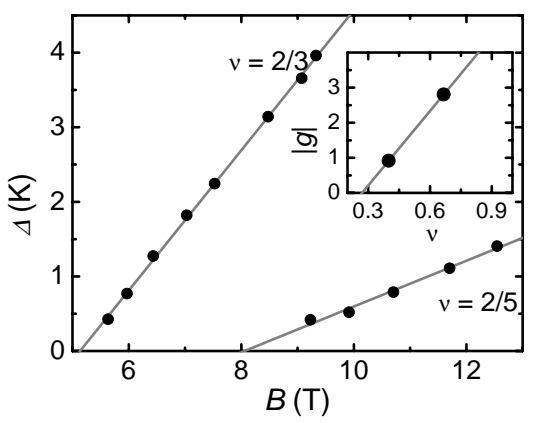

FIGURE 2. Activation gaps $\Delta(B)$ at filling factors $v=2 / 3$ and $v=2 / 5$ as a function of the magnetic field. The activation gaps are nicely fitted by a linear dependence as shown by the solid lines. Inset: $g$-factor extracted from the linear dependence of the activation energy $\Delta$ (see text). The line shows a linear interpolation [14]. expectation. We want to point out that activation gaps measured by transport are typically reduced by disorder and finite thickness effect. This effect is however expected to depend only weakly on $B$. The measured slope $\partial_{B} \Delta(B)$ is therefore essentially unaffected and yields the CF $g$-factor through Eq. (2), producing $\left|g_{2 / 5}\right|=0.92$ and $\left|g_{2 / 3}\right|=2.80$. We apply Eq. (2) in a quite large magnetic field range, nevertheless we only expect 5-10\% deviation from the linear behaviour. Astonishingly, the linearity in the experiment seems to be even better, maybe as a result of slight $B$ dependent finite thickness effect.

The values of $g$ indicate a strong renormalization due to interactions (the bulk $g$-factor for GaAs being -0.44). Moreover, the CF $g$-factor depends strongly on the electronic filling factor. In fact, although both the two fractions $v=2 / 3$ and $2 / 5$ are mapped into the same CFfilling $p=2$ (symmetrically with respect to $v=1 / 2$ ) their $g$-factors differ by more than a factor 3 .

Since our $\mathrm{CF}$ form around $v=1 / 2$ it is interesting to estimate $g_{1 / 2}$. For the exchange enhanced electronic $g$-factor a linear scaling with filling factor is expected from theory [14]. This linear interpolation yields $\left|g_{1 / 2}\right|=$ 1.65 (see inset of Fig. 2), in excellent agreement with $\left|g_{1 / 2}\right|=1.6$ obtained by Kukushkin [4, 8]. In contrast, NMR experiments in tilted fields found $\left|g_{1 / 2}\right| \approx 0.39$ [15].

In conclusion, we performed a direct measurement of the CF $g$-factor for $v=2 / 5$ and $2 / 3$. Although the two fractions are equivalent in the $\mathrm{CF}$ picture, their $g$-factor is significantly different, showing interactionrenormalization and a strong dependence on the electronic filling factor $v$.

We acknowledge financial support by BMBF, TMR, DFG and the Feinberg School of the Weizmann Institute.

\section{REFERENCES}

1. J. P. Eisenstein et al., Phys. Rev. Lett. 62, 1540 (1989).

2. L. W. Engel et al., Phys. Rev. B 45, 3418 (1992).

3. R. R. Du et al., Phys. Rev. Lett. 75, 3926 (1995).

4. I. V. Kukushkin et al., Phys. Rev. Lett. 82, 3665 (1999).

5. J. K. Jain, Phys. Rev. Lett. 63, 199 (1989).

6. X. G. Wu et al., Phys. Rev. Lett. 71, 153 (1993).

7. K. Park and J. K. Jain, Phys. Rev. Lett. 80, 4237 (1998).

8. E. Mariani et al., Ann. Phys. (Leipzig) 11, 926 (2002).

9. F. Schulze-Wischeler et al., Phys. Rev. Lett. 92, 156401 (2004).

10. B. I. Halperin et al., Phys. Rev. B 47, 7312 (1993).

11. G. S. Boebinger et al., Phys. Rev. Lett. 55, 1606 (1985).

12. R. R. Du et al., Phys. Rev. Lett. 70, 2944 (1993).

13. G. Murthy, Phys. Rev. Lett. 84, 350 (2000); E. Mariani et al., Phys. Rev. B 66, 241303(R) (2002).

14. T. Ando et al., Rev. Mod. Phys. 54, 437 (1982).

15. N. Freytag et al., Phys. Rev. Lett. 89, 246804 (2002). 
Copyright of AIP Conference Proceedings is the property of American Institute of Physics. The copyright in an individual article may be maintained by the author in certain cases. Content may not be copied or emailed to multiple sites or posted to a listserv without the copyright holder's express written permission. However, users may print, download, or email articles for individual use. 